\title{
A New Approach to Medical Image Stitching Using Minimum Average Correlation Energy Filter and Peak to Side-Lobe Ratio
}

\author{
S. Salbiah, A. Somaya, H. Arof, Z. S. Saleh, F. Ibrahim
}

\section{INTRODUCTION}

Medical imaging is an important tool in healthcare services for diagnosis, planning, executing, and evaluating surgical and therapeutical procedures. X-rays are the oldest and most frequently used form of medical imaging. Imaging with X-rays involves disclosing a part of the body to a small amount of ionizing radiation to produce images of the inside of the body or part of it (Maintz et al., 1998).

When imaging long parts of a human body (e.g., legs or spine) using conventional screen-film technique, special cassettes and films of limited size are used. To produce images containing the whole body parts, digitized images from the cassettes and films that contain portions of the body parts can be assembled using image stitching. For example, in conventional radiography, a large image can be assembled from X-ray images of multiple exposures with a small spatial overlap ( $C^{`}$ apek et al., 2002; Pluim et al., 2003; Gooßena et al., 2008; Kumar et al., 2010; Wang and Wang, 2010).

Image stitching plays an important role in panorama image creation, super resolution image formation, medical image analysis, and many other computer vision applications (Brown, 1992; Chen, 1998; Maintz et al., 1998; Szeliski, 2006; Gooßena et al., 2008). In an application that uses image stitching, its performance carries a significant impact to the overall efficiency of the application. Basically, image stitching is a process of generating a big image by combining a series of smaller, overlapping subimages (Chen, 1998). It consists of two steps namely image registration and image merging (blending). In image registration, portions of two adjacent or consecutive images are compared to find the merge position and the needed translation that will be used to align the images. Once 
the overlapping images have been registered, the task is to find the optimal spatial and intensity transformations so that the images are matched and merged seamlessly. This process is then repeated by merging the resulting image with the next image until all images are combined into a single image wherein the transitions between adjacent subimages are visually undetectable.

Broadly speaking, automatic image stitching methods fall into two main categories, and they are the direct-based methods and featurebased methods (Brown, 1992; Chen, 1998; Maintz et al., 1998;

Szeliski, 2006). Direct-based methods use pixel to pixel matching to maximize a measure of image similarity between two subimages and subsequently find a parametric transformation to combine the two subimages. Feature-based methods first extract salient features such as corners from the two subimages and then establish reliable feature correspondences by comparing the features. Then images are warped according to parametric transformations that are estimated from those correspondences (Feng et al., 2010). Direct methods have the advantage that they use all of the available image data and hence can provide very accurate registration, but being iterative methods, they require initialization. Unlike direct-based methods, feature-based methods do not require initialization but they are time consuming and for the majority of cases, finding features in subimages is difficult ( $\mathrm{Li}$ and Ma, 2006). Some other methods can be regarded as the combinations of the two above-mentioned methods

(Brown, 1992; Chen, 1998; Maintz et al., 1998; Szeliski, 2006).

Cross correlation is a basic statistical approach used as a similarity measure in many image registration procedures. It is a match metric between two subimages. This similarity measure is widely used as it can be computed efficiently using the fast Fourier transform (FFT) especially for combining large subimages of the same size. Furthermore, both direct correlation and correlation using FFT have costs that grow at least linearly with the image area (Brown, 1992).

Another direct-based approach that uses correlation filters has been applied in automatic target recognition (Mahalanobis et al., 1998) and biometric identification (Savvides, 2004; Riedel et al., 2005). The simplest form of correlation filter is known as the matched spatial filter (MSF) (Lugt, 1964; Kumar et al., 2005). It performs well at detecting a reference image corrupted by additive white noise but this technique suffers from distortion variance, poor generalization, and poor localization properties. The reason for this poor performance is because MSF uses a single training filter to 
generate broad correlation peaks (Riedel et al., 2005). This shortcoming

is addressed by introducing another correlation filter that is

known as a synthetic discriminant function (SDF). It is a linear

combination of MSFs. It linearly combines a set of training images

into one filter, which further allows users to constrain the filter output

at the origin of the correlation filter (Mahalanobis et al., 1987).

These prespecified constraints are known as "peak constraints."

SDF filters provide some degree of distortion in variance but like

MSFs they result in large side-lobes and broad correlation peaks

that make localization difficult.

To reduce large side-lobes observed in SDFs and to maximize peak sharpness for better object localization and detection, MACE

(minimum average correlation energy) filters were introduced. A

MACE filter can be obtained from the FFT of a single training image or synthesized from the FFTs of a few training images.

When the FFT of a test image is presented, a 2D correlation plane

is computed and a sharp correlation peak is observed at the position that produces the maximum correlation between the test image and the training image (Savvides et al., 2002, 2003; Kumar et al., 2006).

For image stitching, the correlation values are very close to zero for all points except at the location where the two subimages match. Exploiting this simple attribute of the MACE filter, a stitching framework based on pattern correlation (PCB) is developed, and its details are presented in the following sections. The proposed system uses correlation filters to find the best matched position for two Xray images that are combined to form a single image.

A. Image Stitching for Medical Imaging. For medical imaging, direct-based methods using the full image content have been widely used and regarded as the methods of choice. Their advantage is due to the fact that they do not reduce the grey-level images to relatively sparse extracted information but use all of the available information throughout the registration process (Maintz et al., 1998;

C `apek et al., 2002; Kumar et al., 2010; Wang and Wang, 2010).

Wang and Wang (2010) adopted the grid-based registration method for infrared medical images. They used the sum of squared difference metric to measure similarity between pixels of the two images. To improve the registration accuracy and reduce computational time, they divided the registration process into two steps. The first step is a rough registration, which records the best registration point position, while the second step is a precise registration, which 
fine-tunes the result of the first step. After locating the best registration point in the first step, the template moves in small steps around the selected point and computes the square of difference of corresponding pixels of the two images in the second step (Wang and

Wang, 2010). This strategy decreases the processing time slightly.

An alternative to take intensity differences is to measure the correlation between the two aligned images (Maintz et al., 1998).

C `apek et al. (2002) used the point matching method together with the normalized correlation coefficient (NCC) to evaluate the similarity of X-ray images. They claimed that their method produces accurate results but the processing time is long $\left(\mathrm{C}^{`}\right.$ apek et al., 2002). The normalized cross correlation (NCC) score is always in the range of $[21,1]$, which makes it easier to be implemented in some higher-level applications. However, the NCC score is undefined if any one of the two patches has zero variance. In fact, its performance degrades for noisy low-contrast regions.

In this article, a robust method is proposed for medical image stitching. The method uses MACE filter and peak to side-lobe ratio (PSR) as a similarity measure. The rest of this article is organized as follows. Section II describes the algorithms used in every step of the system. The experiments and their results are discussed in section III, and a conclusion is drawn in section IV.

\section{IMAGE STITCHING SYSTEM}

The proposed pattern correlation-based (PCB) image stitching system is shown in Figure 1. It consists of seven steps which are preprocessing, windowing, Fourier transformation, correlation calculation, inverse transformation, peak and PSR measurement, and image blending. In the forthcoming sections, details of each step are discussed.

\section{A. Preprocessing and Windowing. Two databases of X-ray} images are used for this work. The first one is a custom database of spine images obtained from a local hospital, and the second database consists of open-access images acquired from the Internet.

From each database, two images are selected as inputs to the system at a time. The system will decide whether the input images are overlapping and how to merge them. Otherwise, no action will be taken. During the preprocessing step, all images are histogram equalized to remove intensity variations caused by external factors such as uneven illumination. Then windowing is performed using Hanning window before Fourier transformation to avoid leakage in 
the signal caused by its energy smearing out over a wide frequency range in the FFT when it should be in a narrow frequency range (LDS Group, 2011, www.lds-group.com).

Full text is available at :

http://onlinelibrary.wiley.com/doi/10.1002/ima.22018/full 